\title{
Monitoring Cardiopulmonary Resuscitation Quality in Emergency Department: A National Survey in China on Current Knowledge, Attitudes, and Practices
}

\section{Kang Zheng}

Peking University Third Hospital

Lanfang Du

Peking University Third Hospital

\section{Yu Cao}

West China Hospital of Sichuan University

Zhendong Niu

West China Hospital of Sichuan University

\section{Zhenju Song}

Zhongshan Hospital Fudan University

Zhi Liu

First Hospital of China Medical University

Xiaowei Liu

First Hospital of China Medical University

\section{Xudong Xiang}

Second Xiangya Hospital of Central South University

\section{Qidi Zhou}

Peking University Shenzhen Hospital

\section{Hui Xiong}

Peking University First Hospital

\section{Fengying Chen}

The Affiliated Hospital of Innor Mongolia Medical University

\section{Guoqiang Zhang}

China-Japan Friendship Hospital

Qingbian Ma ( $\triangle$ maqingbian@medmail.com.cn )

Peking University Third Hospital

\section{Research Article}


Keywords: Cardiac arrest, cardiopulmonary resuscitation, high-quality cardiopulmonary resuscitation, cardiopulmonary resuscitation quality control.

Posted Date: October 8th, 2021

DOI: https://doi.org/10.21203/rs.3.rs-952645/v1

License: (c) (1) This work is licensed under a Creative Commons Attribution 4.0 International License. Read Full License

Version of Record: A version of this preprint was published at BMC Emergency Medicine on February 28th, 2022. See the published version at https://doi.org/10.1186/s12873-022-00590-z. 


\section{Abstract}

Background: To investigate current knowledge, attitudes, and practices for CPR quality control among emergency physicians in Chinese tertiary hospitals.

Methods: Anonymous questionnaires were distributed to physicians in 75 tertiary hospitals in China between January and July 2018.

Results: A total of 1405 respondents answered survey without obvious logical errors. Only $54.4 \%$ knew all criteria of high-quality CPR. The main problems during CPR were related to chest compression: low quality due to fatigue (67.3\%), inappropriate depth (57.3\%) and rate (54.1\%). 91.0\% respondents considered CPR quality monitoring should be used, $72.4 \%$ knew objective method for monitoring, and 63.2\% always/often monitored CPR quality during actual resuscitation. The utilization rate of recommended monitoring methods was reported as follow, $\mathrm{ETCO}_{2}$ was $42.7 \%$, audio-visual feedback devices was $10.1 \%$, coronary perfusion pressure was $17.9 \%$, and invasive arterial pressure was $31.1 \%$. 96.3\% respondents considered it was necessary to participate in regular CPR retraining, but $21.4 \%$ did not receive any retraining. The ideal retraining interval was considered to be 3 to 6 months, but actual interval was 6 to 12 months. CPR feedback devices were always/often used in training was reported by $49.7 \%$ of respondents.

Conclusion: Chinese emergency physicians were very concerned about CPR quality, but they did not fully understand the high-quality criteria and impact on prognosis. CPR quality monitoring was not routine procedure during actual resuscitation. The methods recommended in guidelines were rarely used in practice. Many physicians have not received retraining or have received retraining at long intervals. Feedback devices were not commonly used in training.

\section{Background}

The outcomes of cardiac arrest patients in China have been very poor. In Beijing, the capital of China and where medical technology was well developed, Only $1.3 \%$ out-of-hospital cardiac arrest (OHCA) patients were discharged, and $1.0 \%$ survived with a good neurological outcome (defined as cerebral performance category score was 1 or 2 ) in $2012^{[1]} .9 .1 \%$ in-hospital cardiac arrest (IHCA) patients were discharged and $6.4 \%$ survived with a good neurological outcome in $2014^{[2]}$.

Cardiopulmonary resuscitation (CPR) was a lifesaving intervention and the cornerstone of resuscitation from cardiac arrest. When cardiac arrest was taking place, blood circulation was wholly stopped. CPR could provided $10 \%$ to $30 \%$ of normal blood flow to the heart and $30 \%$ to $40 \%$ of normal blood flow to the brain $^{[3]}$. Early CPR was an important link in the survival chain, no matter in OHCA or IHCA patients ${ }^{[4]}$.

Survival from cardiac arrest depended on early recognition of the arrest event and immediate activation of the emergency response system, but equally critical was that CPR delivered was high-quality. Highquality CPR included ensuring adequate chest compression rate and depth, allowing full chest recoil 
between compressions, minimizing interruptions in chest compressions, and avoiding excessive ventilation $^{[5]}$. There was clear evidence that providing high-quality CPR significantly improves resuscitation outcomes ${ }^{[6-9]}$.

Poor-quality CPR should be considered preventable harm. Monitoring both patient physiologic parameters and provider performance during CPR was essential to optimizing CPR quality ${ }^{[5]}$. Visual observation was the most basic monitoring method. In 2013, the American Heart Association (AHA) published a Consensus Statement focused on strategies to improve CPR quality ${ }^{[9]}$. Patient physiologic parameters during CPR that were considered pertinent for monitoring included invasive hemodynamic data (coronary perfusion pressure $>20 \mathrm{mmHg}$ or arterial diastolic pressure $>25$ $\mathrm{mmHg}$ ) and end-tidal carbon dioxide $\left(\mathrm{ETCO}_{2}\right)$ concentrations $>20 \mathrm{mmHg}^{[9]}$. Monitors with audiovisual feedback devices to measure provider CPR performance were widely accepted ${ }^{[9]}$. In 2015, the AHA's CPR guidelines recommended using feedback devices for real-time optimization of CPR performance ${ }^{[5]}$.

Today, few healthcare organizations consistently applied strategies of systematically monitoring CPR quality even though there was an unacceptable disparity in the quality of resuscitation care and outcomes $^{[5]}$. As the current knowledge, attitudes, and practices for CPR quality monitoring in China have never been reported, the aim of the present survey was to investigate the use of monitoring among Chinese emergency physicians.

\section{Methods}

\subsection{Geographical background}

Mainland China was divided into six administrative regions according to geographical distribution, including the east region, north region, northeast region, southwest region, south-central region, and northwest region. These regions differed in medical development ${ }^{[10]}$.

\subsection{Study design}

This was a cross-sectional multicenter study. In China, only tertiary, or Level III, hospitals had independent emergency departments and were also teaching hospitals, so these hospitals were identified in an attempt to target providers most likely to care for cardiac arrest patients. Tertiary general hospitals in mainland China were selected by separate random sampling in the six administrative regions. We screened for all 374 tertiary general hospitals, which were then coded randomly by SPSS version 25.0 (IBMCorp, Armonk, New York, USA) and then were arranged in ascending order in each administrative region. The top $20 \%$ of hospitals were enrolled in the study, with alternative hospitals considered (in ascending order) if any enrolled hospitals were inaccessible or refused to participate. Finally, 75 hospitals were included. We then conducted a questionnaire survey for all emergency physicians of the selected hospitals. 
The questionnaire was developed by a senior emergency physician, and examined and discussed three times by an expert team consisting of an epidemiologist and emergency specialists experienced in the management of cardiac arrest patients. The questionnaire included three parts: (1) background data of respondent; (2) the respondent's awareness and practices for quality monitoring during actual CPR on a patient; (3) and awareness and practices for quality monitoring in CPR training. A total of 30 questions were included in the questionnaire, including 3 blank questions, 20 single choice questions and 7 multiple choice questions. The paper questionnaires were distributed to the directors of selected emergency departments by mail from January to March in 2018. Data collection ended in July 2018.

The study protocol was approved by Peking University Third Hospital Medical Science Research Ethics Committee (Project number: IRB00006761-M2018030, Ethics approval document number: 2018-176-01).

\subsection{Statistical methods}

The data were analyzed by SPSS version 25.0 (IBMCorp, Armonk, New York, USA). Quantitative variables were expressed as mean (standard deviation) when following a Gaussian distribution, or median (interquartile range $25 \%-75 \%$ ) otherwise. Qualitative variables were expressed as frequencies.

\section{Results}

A total of 1489 (93.1\%) responses were collected from 1600 questionnaires in 75 hospitals; 1405 responses were analyzed and 84 were excluded because of obvious logical errors. General characteristics of respondents were described in Table 1.

Table 1 Characteristics of respondents 
Characteristics

$\mathrm{n}$

Gender

Male

Female

No answer

Age (years), mean $\pm S D$ *

Academic degrees

Bachelor

Master

Doctorate

No answer

Title

Resident

Attending

Associate chief physician

Chief physician

No answer

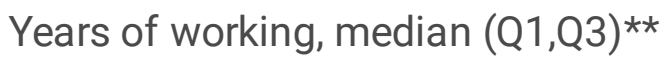

Hospital location

East region

North region

Northeast region

South-central region

Southwest region

Northwest region

Number of treated cardiac arrest patients per year

$0-10$

$315 \quad$ (22.4)

11-30

507

251

(57.8)

$576 \quad$ (41.0)

17

$35.28 \pm 7.40$

532

$694 \quad$ (49.4)

146 (10.4)

33

611 (43.5)

$500 \quad$ (35.6)

209 (14.9)

$64 \quad$ (4.6)

21

$7(3,13)$

$290 \quad$ (20.6)

$247 \quad$ (17.6)

209 (14.9)

$335 \quad$ (23.8)

199 (14.2)

125

$31-50$ 


\begin{tabular}{|c|c|c|}
\hline$>50$ & 320 & $(22.8)$ \\
\hline No answer & 12 & $(0.9)$ \\
\hline \multicolumn{3}{|c|}{ ROSC in treated cardiac arrest patinets } \\
\hline$>30 \%$ & 302 & $(21.5)$ \\
\hline $21 \%-30 \%$ & 303 & $(21.6)$ \\
\hline $11 \%-21 \%$ & 246 & $(17.5)$ \\
\hline $6 \%-10 \%$ & 265 & $(18.9)$ \\
\hline$<6 \%$ & 274 & $(19.5)$ \\
\hline No answer & 15 & $(1.1)$ \\
\hline \multicolumn{3}{|c|}{ Discharge survival in treated cardiac arrest patinets } \\
\hline$>30 \%$ & 137 & $(9.8)$ \\
\hline $21 \%-30 \%$ & 176 & $(12.5)$ \\
\hline $11 \%-21 \%$ & 228 & $(16.2)$ \\
\hline $6 \%-10 \%$ & 311 & $(22.1)$ \\
\hline$<6 \%$ & 538 & $(38.3)$ \\
\hline No answer & 15 & $(1.1)$ \\
\hline \multicolumn{3}{|c|}{ Good neurological outcome in treated cardiac arrest patinets } \\
\hline$>30 \%$ & 125 & $(8.9)$ \\
\hline $21 \%-30 \%$ & 153 & $(10.9)$ \\
\hline $11 \%-21 \%$ & 181 & $(12.9)$ \\
\hline $6 \%-10 \%$ & 286 & $(20.4)$ \\
\hline$<6 \%$ & 644 & $(45.8)$ \\
\hline No answer & 16 & $(1.1)$ \\
\hline
\end{tabular}

\subsection{Knowledge and attitudefor high-quality CPR and quality monitoring}

The reported knowledge and attitude for CPR quality and quality monitoring were described in Table 2. 
Table 2 Knowledge and attitude for CPR quality and quality monitoring 
Which of the following criterion are included in high quality CPR? $(n=1402)$

ensuring adequate chest compression depth

ensuring adequate chest compression rate

allowing full chest recoil between compressions

1261

minimizing interruptions in chest compressions

956

ensuring adequate rate of ventilation

1209

ensuring adequate volume of ventilation

Should CPR quality be monitored during resuscitation? $(n=1387)$

Yes

1278

No

63

I don't know

46

Should CPR quality be monitored during resuscitation, when use mechanical CPR device? $(n=1376)$

Yes

1298

(94.3)

No

40

I don't know

38

Whether a dedicated person is responsible for quality monitoring? $(n=1389)$

Yes

1220

No

91

I don't know

78

Do you know any objective method or technology of CPR quality monitoring? $(n=1400)$

Yes

No

How do you learn about monitoring mothed or technique? $(n=1014)$

Clinical guideline

Academic conference

Medical literature 
Do you know which of the following quality monitoring methods ? ( $n=1014)$

Palpation of arterial pulse

Observing of the ECG waveform

Observing of the SpO2 waveform

Pulse oximetry plethysmographic waveform 484

End-Tidal CO2

727

Coronary perfusion pressure

Invasive arterial pressure 566

Audiovisual feedback device

Others

Do you think CPR quality monitoring could improve CPR quality? ( $n=1394)$

Yes

No

29

No clear conclusion

120

Don't know relevant research

87

Do you think CPR quality monitoring could improve the return of spontaneous circulation? $(n=1391)$

Yes

1094

(78.6)

No

38

No clear conclusion

158

Don't know relevant research

101

Do you think CPR quality monitoring could improve survival? ( $n=1374)$

Yes

1024

No

36

No clear conclusion

195

Don't know relevant research

119

Do you think CPR quality monitoring could improve the neurologic outcome? $(n=1393)$ 
$54.4 \%$ of respondents knew all six criteria; $60.9 \%$ knew all four criteria of high-quality chest compression and $78.2 \%$ knew both criteria of avoiding excessive ventilation.

\subsection{The Practices of CPR quality monitoring during actual CPR}

The reported practices of CPR quality monitoring during actual resuscitation were described in Table 3 .

Table 3 The practices of CPR quality monitoring during actual resuscitation 
What are the common problems during actual resuscitation? $(n=1364)$

Low quality of chest compression due to fatigue

Can not ensure appropriate chest compression depth

Can not ensure appropriate chest compression rate

No full chest recoil between compressions

706

Long interruptions between chest compressions 684

Can not ensure appropriate ventilation rate

Can not ensure appropriate ventilation volume 667

Too short ventilation delivery time 643

Insufficient capacity of team leader

Poor cooperation between team members

Others

Do you use mechanical CPR devices during actual resuscitation? ( $n=1391)$

Always

Often

407

Sometimes

335

Rarely

155

Never

327

Which type of mechanical CPR devices do you use? $(n=1038)$

Piston device only

770

Load-distributing band device only

81

Both of above

152

Others

Do you monitored CPR quality during actual resuscitation? $(n=1375)$

\section{Always}

485

Often

Sometimes

210

Rarely

79 
Which method do you use for monitoring quality ? $(n=1158)$

Palpation of arterial pulse

Observing of the ECG waveform

994

Observing of the SpO2 waveform

811

(70.0)

Pulse oximetry plethysmographic waveform

274

End-Tidal CO2

495

Coronary perfusion pressure

207

Invasive arterial pressure

360

Audiovisual feedback device

117

(10.1)

Others

6

Does the method you used could achieve the monitoring purposes? $(n=1158)$

Completely

Most

528

Few

496

Never

50

What is the interval from the start of CPR to start of quality monitoring? $(n=1149)$

0-2minutes

496

3-6minutes

471

7-10minutes

100

$>10$ minutes

82

Is there a dedicated person responsible for monitoring during actual resuscitation? $(n=1152)$

Always

Often

Sometimes

Rarely

Never
255

299

289

210

99

Use of recommended monitoring technology and reasons for not always using were described in Fig. 1 and Fig. 2. 


\subsection{Quality monitoring in CPR training}

Practices and attitudes on quality monitoring in CPR training were described in Table 4.

Table 4 Quality monitoring in CPR training 
Training and retraining

Do you think emergency physicians should participate in regular CPR retraining? $(n=1346)$

Yes

No

Do not know

what is the ideal interval between retraining in your opinion? $(n=1294)$

3 months

6 months

500

12 months

272

24 months

71

Other interval

8

Have you attend CPR training or retraining as a trainee? $(n=1344)$

Yes

No

What is the ideal interval between your retraining in actual? $(n=1043)$

3 months

283

6 months

332

12 months

333

24 months

79

Other interval

16

CPR feedback devices

Do you think using CPR feedback devices could improve CPR performance in training? $(n=1343)$

Yes

1210

No

45

Do not know

88

Did you used CPR feedback devices in your actual training or retraining? $(n=1052)$ 
which part of training dos CPR feedback devices usually be used? $(n=787)$

Skill training

Practice

Test

\section{Discussion}

\subsection{Knowledge and attitudes for CPR quality monitoring}

We found it was a good situation that $\mathbf{9 2 . 1 \%}$ of respondents considered quality monitoring was needed during CPR. Mechanical compression devices were designed for some special situations and were considered to reduce the physical burden of emergency physicians ${ }^{[11]}$. Because the emergency department in China was often crowded, the use rate of mechanical devices during resuscitation was high. In this situation, $94.3 \%$ of respondents considered CPR quality need to be monitored. This showed that emergency physicians were concerned about the CPR quality monitoring.

High-quality CPR was an important link between survival chains, and it may be more important than other links ${ }^{[4]}$. Unfortunately, only $\mathbf{5 4 . 4 \%}$ of respondents to this survey knew all six criteria of high-quality CPR and $60.9 \%$ knew all four criteria of high-quality chest compression. Most respondents were concerned about depth, rate and chest recoil during compression. But it was concluded that emergency physicians in tertiary general hospitals did not pay enough attention to minimizing interruptions. Minimizing interruption was also an important criterion of high-quality CPR emphasized in AHA's CPR guidelines ${ }^{[5]}$. Continuous chest compressions could maintain adequate coronary perfusion pressure, thereby increasing the likelihood of achieving ROSC ${ }^{[12]}$. Chest compression fraction of $80 \%$ was recommended to make sure compressions were continued with as few interruptions as possible during CPR. But in this survey, only $68.0 \%$ of respondents knew this criterion. This was a problem for improving CPR quality and it was important to strengthen education on minimizing interruption.

Visual observation was the most basic and widely used monitoring method, but objective parameters were recommended to be used for monitoring ${ }^{[9]}$. We found $72.4 \%$ of respondents knew objective monitoring technique. Clinical guidelines and academic conference were the main way to understand the technology. But in our survey, Chinese emergency physicians did not know much about the techniques recommended in the guidelines, especially Audiovisual feedback devices. 
It was important to note that respondents revealed some misunderstandings regarding clinical findings on CPR quality control. For example, $71.3 \%$ of Chinese emergency physicians considered that using CPR quality monitoring devices could improve cardiac patient's outcome. While current studies have not demonstrated significant improvement in outcome related to CPR quality monitoring during actual cardiac arrest events ${ }^{[5,9,13]}$.

Although Chinese emergency physicians were concerned about CPR quality, they were lack of understanding of few criteria, new objective parameter monitoring methods and research results.

\subsection{Practices in CPR quality monitoring}

Chest compression quality was considered by respondents to be a main problem during actual resuscitation in China. Low quality due to fatigue, inappropriate compression depth, and inappropriate compression rate were the top three problems noted in actual CPR. In contrast, personal ability and cooperation of resuscitation team members were not issues. Because of this, quality control was placed on a vital position in China. But CPR quality monitoring was not shown to be a routine procedure in Chinese emergency department. Only $63.2 \%$ of respondents replied that they always/often monitored CPR quality during actual resuscitation. This showed that in clinical practice, the compliance of Chinese emergency physicians in CPR quality monitoring was not high.

Accurate measurement of CPR quality was precondition for high quality CPR. Objective parameter monitoring methods were better than visual observation ${ }^{[5]}$. Our results showed that recommended monitoring methods were rarely used in Chinese emergency department. $\mathrm{ETCO}_{2}$ was the most commonly used methods. The opinions and clinical experience of experts strongly supported prioritizing the use of $\mathrm{ETCO}_{2}$ to adjust the compression technique during resuscitation ${ }^{[5,9]}$. Endotracheal intubation was not difficult for emergency physicians, because of wide use of advanced equipment like videolaryngoscope in China. Previous studies have shown that advanced airway could be placed in the first few minutes during resuscitation ${ }^{[14]}$. In this situation, ETCO2 data was easier to obtain. This may be the reason of why ETCO2 was widely utilized in China. However, audio-visual feedback devices was another method recommended by AHA's CPR guidelines for quality monitoring ${ }^{[5]}$. It was a noninvasive technology for realtime monitoring, recording, and feedback about CPR performance ${ }^{[15,16]}$. We found that less than $20 \%$ of respondents have used this technology. Pulse oximetry was a common clinical technique, its waveform could reflect peripheral tissue perfusion. Some research found that appearance of pulse oximetry plethysmographic waveform was related to CPR quality $[17,18]$. Pulse oximetry plethysmographic waveform, as a monitoring technology, was recommended for CPR quality monitoring by Chinese expert consensus in 2018. However, the utilization rate of this technology was still lower than that of $\mathrm{ETCO}_{2}$ and Invasive arterial pressure. Lack of equipment and understanding were the main reasons respondents gave for the low usage rate in China.

The biggest problem in quality monitoring was that unrecommended methods were widely used for quality monitoring in Chinese emergency department. Palpation of the arterial pulse, observing ECG 
waveform, and observing $\mathrm{SpO}_{2}$ waveform were the top three most commonly used method. Palpation of arterial pulse was the most commonly used method to evaluate chest compression quality; but it has been shown to be unreliable and cannot be used for continuous monitoring during actual resuscitation ${ }^{[19}$, 20]. Therefore, the guidelines did not recommend this method for CPR quality monitoring, ${ }^{[9]}$. Regular ECG waveforms accompanying chest compression can be observed in some patients, but shape of waveform has no clear relationships with the quality of chest compressions ${ }^{[21]}$. ECG waveform was widely used for quality monitorin, that reflected the misunderstanding of its meaning in Chinese emergency physicians.

Continuing education of physicians in emergency departments was crucial to increase the rates of utilization of recommended technology ${ }^{[22]}$. Although $\mathrm{CPR}$ quality monitoring has been recommended by CPR guidelines, few specific consensus protocols exist that provide detail on how to best implement the monitoring. The development and publication of standardized monitoring protocols will likely help physicians to better utilize CPR quality monitoring in China.

\subsection{Quality monitoring in CPR training}

Basic life support and advanced cardiac life support techniques were the core skills of resuscitation ${ }^{[23]}$. CPR training course was a key part of Chinese resident training program. In most tertiary hospitals, all emergency physicians needed to take basic life support courses and many of them also needed to take advanced cardiac life support courses. CPR training courses in many hospitals were certified by the American Heart Association ${ }^{[24]}$.

CPR training was not a one-time training. Retraining was recommended by AHA guidelines, because skills and knowledge may decay within 3 to 12 months after initial training ${ }^{[23]}$. The retraining system was widely accepted in China, where $96.3 \%$ of respondents believed it was necessary to retrain after initial training. Responses showed that CPR retraining received great attention, and that a "frequent" training system was more acceptable. $72.8 \%$ of respondents considered the ideal interval between trainings to be 3 to 6 months, though there was no clear recommendation on optimum time interval ${ }^{[23]}$. Unfortunately, the survey results revealed a large gap between ideal training programs and actual implementation, as $21.4 \%$ of emergency physicians did not receive any retraining after initial training. Among those who did, the actual retraining interval was 6 to 12 months, significantly longer than desired. This showed that most hospitals did not have standard retraining systems. Retraining may be difficult to implement in some hospitals. Short-frequent retraining may be a solution in China. Because it would not increase the cost, if total training time was fixed. Physicians were more likely to take part in short time training course after busy work. And frequent retraining was helpful to the consolidation of skills.

Chinese physicians were in agreement on the use of new technology and equipment for CPR training. According to AHA's CPR guidelines, feedback devices should be used in training to learn CPR skills ${ }^{[23]}$. This attitude was supported by $90.1 \%$ of respondents, who believed that CPR feedback devices can improve performance during training. However, attitudes and practice was so different. The typical training course in Chinese hospitals includes two parts: theoretical teaching and simulation exercises. 
While the structure of the training course is reasonable, only $49.7 \%$ of respondents replied that CPR feedback devices were always/often used in training, and $25.1 \%$ replied that they never used feedback devices. In developing countries like China, there were many hospitals that have no ability to purchase these devices. This may become an important barrier to improving CPR quality.

\section{Conclusions}

In this survey we found Chinese physicians in tertiary hospitals were very concerned about CPR quality, but they did not fully understand the high-quality criteria and impact on prognosis. Most emergency physicians considered it was necessary to monitor CPR quality, but quality monitoring was not routine procedure during actual resuscitation. Recommended monitoring methods such as audio-visual feedback device was rarely used in practice. However, many physicians used methods not recommended. Although retraining was considered importand, many physicians have not received retraining or have received retraining at long intervals. Feedback devices were not commonly used in training.

In the future Chinese emergency physicians should receive systematic continuing education on CPR quality control. Standard Operation Procedure should be established to guide CPR quality monitoring during actual resuscitation, including hemodynamic parameters, $\mathrm{ETCO}_{2}$ and audio-visual feedback devices. Retraining plan and feedback devices should also be an important part of CPR training.

\section{Limitations}

Emergency department of tertiary hospitals were targeted for this survey, because their providers were likely to have the most experience in caring for cardiac arrest patients. At the same time their providers have a higher level of technology than the others. The overrepresentation of these hospitals may have subjected the survey to bias. In other words, it was likely that the CPR quality monitoring responses reflected in our survey demonstrated an optimistic perspective. The true proportions of knowledge, attitudes, and practices of monitoring across all Chinese hospitals may be lower than those reported in this survey. Additionally, though the majority of published examples of quantifying qualitative data used dichotomous variables for simplicity, such conversion may result in overestimation or underestimation due to identical grading of responses such as "usually" and "always," and "sometimes" and "never."

\section{List Of Abbreviations}

OHCA out-of-hospital cardiac arrest

IHCA in-hospital cardiac arrest

CPR cardiopulmonary resuscitation 
AHA American Heart Association

$\mathrm{ETCO}_{2}$ end-tidal carbon dioxide

\section{Declarations}

Ethics approval and consent to participate

This study was conducted after receiving research ethics approval from the Ethics Committee of Peking University Third Hospital. All participants signed written informed consent before participating in the survey.

Consent for publication

Not applicable.

Availability of data and materials

The datasets used and/or analysed during the current study are available from the corresponding author on reasonable request.

Competing interests

The authors declare that they have no competing interests

Funding

Not applicable.

Authors' contributions

Kang Zheng and Lanfang Du have made contributions to the conception and design of the study, acquisition of data, analysis and interpretation of data, drafting the article and final approval of the version, they contributed equally to this paper.

Yu Cao, Zhendong Niu, Zhenju Song, Zhi Liu, Xiaowei Liu, Xudong Xiang, Qidi Zhou, Hui Xiong, Fengying Chen, as head of each region, have made important contributions to questionnaire modification, regional coordination, data collection, analysis and quality control, revising paper critically for important intellectual content.

Guoqiang Zhang and Qingbian Ma have made contributions to the conception and design of the study, questionnaire modification, regional coordination, acquisition of data, analysis and interpretation of data, revising it critically for important intellectual content.

All authors have read and approved the manuscript. 
Acknowledgements

Not applicable.

\section{References}

1 Shao F, Li CS, Liang LR, Li D, Ma SK. Outcome of out-of-hospital cardiac arrests in Beijing, China. Resuscitation. 2014;85(11):1411-7.

2 Shao F, Li CS, Liang LR, Qin J, Ding N, Fu Y, et al. Incidence and outcome of adult in-hospital cardiac arrest in Beijing, China. Resuscitation. 2016;102:51-6.

3 Halperin HR, Tsitlik JE, Guerci AD, Mellits ED, Levin HR, Shi AY, et al. Determinants of blood flow to vital organs during cardiopulmonary resuscitation in dogs. Circulation. 1986;73(3):539-50.

4 Deakin CD. The chain of survival: Not all links are equal. Resuscitation. 2018;126:80-2.

$5 \quad$ Kleinman ME, Brennan EE, Goldberger ZD, Swor RA, Terry M, Bobrow BJ, et al. Part 5: Adult Basic Life Support and Cardiopulmonary Resuscitation Quality: 2015 American Heart Association Guidelines Update for Cardiopulmonary Resuscitation and Emergency Cardiovascular Care. Circulation. 2015;132(18 Suppl 2):S414-35.

6 Vadeboncoeur T, Stolz U, Panchal A, Silver A, Venuti M, Tobin J, et al. Chest compression depth and survival in out-of-hospital cardiac arrest. Resuscitation. 2014;85(2):182-8.

7 Bossaert L, Van Hoeyweghen R. Evaluation of cardiopulmonary resuscitation (CPR) techniques. The Cerebral Resuscitation Study Group. Resuscitation. 1989;17 Suppl:S99-109; discussion S199-206.

8 Gallagher EJ, Lombardi G, Gennis P. Effectiveness of bystander cardiopulmonary resuscitation and survival following out-of-hospital cardiac arrest. JAMA. 1995;274(24):1922-5.

9 Meaney PA, Bobrow BJ, Mancini ME, Christenson J, de Caen AR, Bhanji F, et al. Cardiopulmonary resuscitation quality: [corrected] improving cardiac resuscitation outcomes both inside and outside the hospital: a consensus statement from the American Heart Association. Circulation. 2013;128(4):417-35.

10 Bekgöz B, Şan İ, Ergin M. Quality Comparison of the Manual Chest Compression and the Mechanical Chest Compression During Difficult Transport Conditions. J Emerg Med. 2020;58(3):432-8.

11 Wik L. Automatic and manual mechanical external chest compression devices for cardiopulmonary resuscitation. Resuscitation. 2000;47(1):7-25.

12 Cunningham LM, Mattu A, O'Connor RE, Brady WJ. Cardiopulmonary resuscitation for cardiac arrest: the importance of uninterrupted chest compressions in cardiac arrest resuscitation. Am J Emerg Med. 2012;30(8):1630-8. 
13 Stiell IG, Brown SP, Christenson J, Cheskes S, Nichol G, Powell J, et al. What is the role of chest compression depth during out-of-hospital cardiac arrest resuscitation. Crit Care Med. 2012;40(4):1192-8.

14 Ge Z, Xia Z, Ma K, Cao J, Mao S, Gong L. [Clinical practice and evaluation of management of patients based on clinical pathway of emergency respiratory and cardiac arrest]. Zhonghua Wei Zhong Bing Ji Jiu Yi Xue. 2019;31(3):313-8.

15 Plata C, Stolz M, Warnecke T, Steinhauser S, Hinkelbein J, Wetsch WA, et al. Using a smartphone application (PocketCPR) to determine CPR quality in a bystander CPR scenario - A manikin trial. Resuscitation. 2019;137:87-93.

16 Zhou XL, Wang J, Jin XQ, Zhao Y, Liu RL, Jiang C. Quality retention of chest compression after repetitive practices with or without feedback devices: A randomized manikin study. Am J Emerg Med. 2020;38(1):73-8.

17 Li C, Xu J, Han F, Zheng L, Fu Y, Yao D, et al. [The role of pulse oximetry plethysmographic waveform monitoring as a marker of restoration of spontaneous circulation:a pilot study]. Zhonghua Wei Zhong Bing Ji Jiu Yi Xue. 2015;27(3):203-8.

18 Xu J, Li C, Zheng L, Han F, Li Y, Walline J, et al. Pulse Oximetry: A Non-Invasive, Novel Marker for the Quality of Chest Compressions in Porcine Models of Cardiac Arrest. PLoS One. 2015;10(10):e0139707.

19 Eberle B, Dick WF, Schneider T, Wisser G, Doetsch S, Tzanova I. Checking the carotid pulse check: diagnostic accuracy of first responders in patients with and without a pulse. Resuscitation. 1996;33(2):107-16.

20 Berg RA, Hemphill R, Abella BS, Aufderheide TP, Cave DM, Hazinski MF, et al. Part 5: adult basic life support: 2010 American Heart Association Guidelines for Cardiopulmonary Resuscitation and Emergency Cardiovascular Care. Circulation. 2010;122(18 Suppl 3):S685-705.

21 Hu Y, Xu J, Yu X. [Rhythm analysis in CPR]. Zhonghua Wei Zhong Bing Ji Jiu Yi Xue. 2017;29(10):946-9.

22 Wright $\mathrm{N}$, Lin $\mathrm{Y}$, Cheng $\mathrm{A}$. How is quality of cardiopulmonary resuscitation being assessed? A national survey of Canadian emergency medicine physicians. CJEM. 2019;21(6):744-8.

23 Bhanji F, Donoghue AJ, Wolff MS, Flores GE, Halamek LP, Berman JM, et al. Part 14: Education: 2015 American Heart Association Guidelines Update for Cardiopulmonary Resuscitation and Emergency Cardiovascular Care. Circulation. 2015;132(18 Suppl 2):S561-73.

24 Cardiopulmonary Resuscitation Specialized Committee of Chinese Research Hospital Association, the Science Popularization Branch of the Chinese Medical Association, Wang L, Meng Q, Yu T. [2018 
National consensus on cardiopulmonary resuscitation training in China]. Zhonghua Wei Zhong Bing Ji Jiu Yi Xue. 2018;30(5):385-400.

\section{Figures}

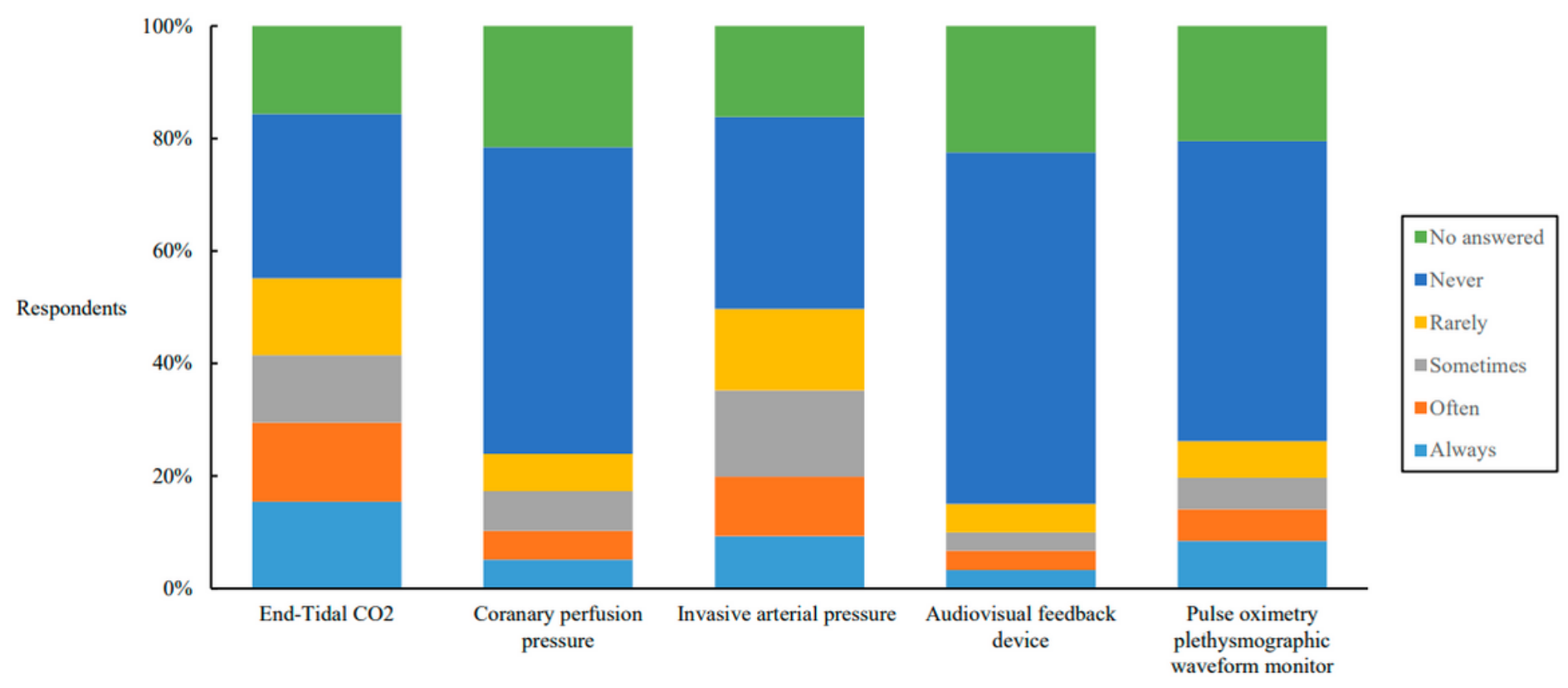

Figure 1

Usage of recommended monitoring technology

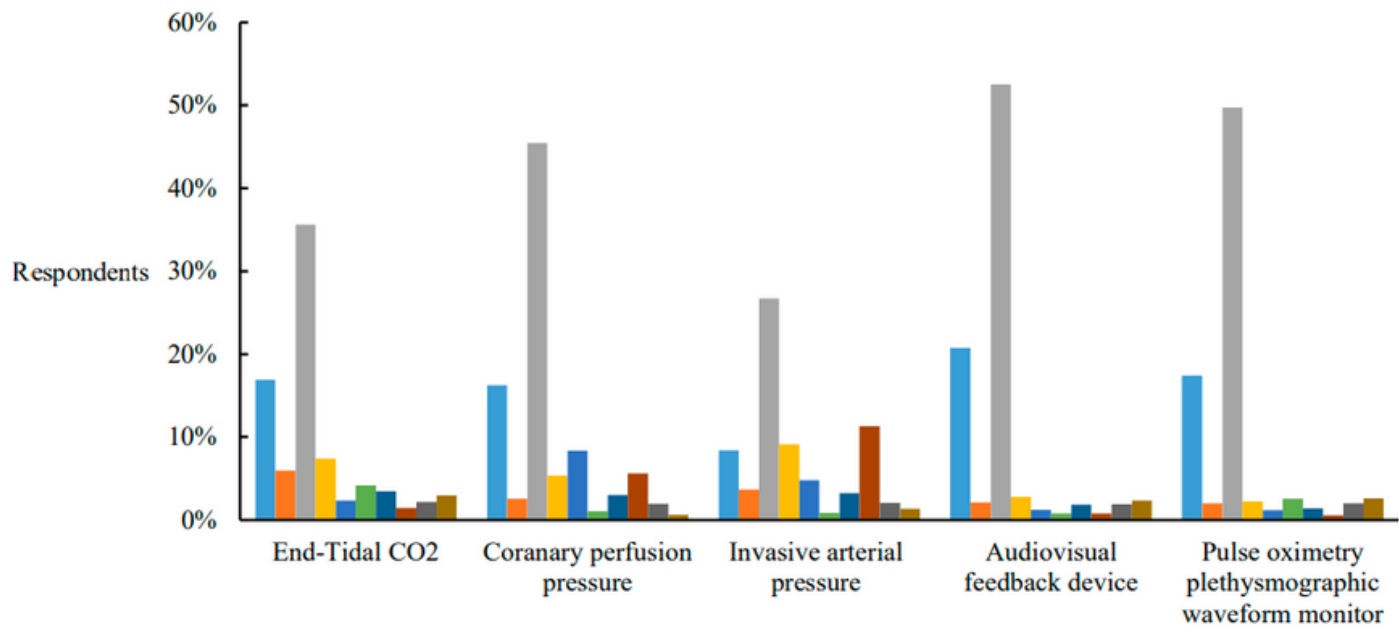

- Do not know this technology

- Insufficient clinical evidence

No equipmen in emergency department

$\square$ Inconvenient to install or use

Difficult to operate

— Low reliability

- Too expensive

- Invasive technology

- Worried about complications

- Others

Figure 2

Reasons for not always using recommended monitoring technology 